\title{
Internet enhanced teleoperation: \\ Toward a remote supervised delay regulator
}

\author{
D. Andreu, P. Fraisse \\ LIRMM - UMR 5506 Université Montpellier II / CNRS \\ 161 rue Ada - 34392 Montpellier CEDEX 5 - FRANCE
}

Submitted for presentation at the 2003 IEEE International Conference on Industrial Technology to be held in Maribor, Slovenia, December 10-12, 2003.

Topics of Interest : Teleoperation

Keywords: Teleoperation, Supervisory control, events, Internet, Telerobotics

Contact : Dr. David Andreu

Tel: (+33) 467418515

Fax: $(+33) 467418500$

e-mail: andreu@lirmm.fr 


\title{
Internet enhanced teleoperation: Toward a remote supervised delay regulator
}

\begin{abstract}
Summary
The Internet is not only an information super highway, but also a means to remotely control systems, as complex robotic devices for instance. A mobile manipulator able to operate in an hazardous and/or dangerous environments can so be used to replace the operator when his intervention would have been difficult, unsafe or impossible. A typical example of use of such a terrestrial mobile manipulator is the handling of an explosive charge in building sites. The Internet is so a way to enhance the human reachability. However for these remote control services to be reliable, one of the important challenges to overcome is the impact of random time delays (when not using dedicated links) on task synchronization, control stability and operator perception of the remote system state.
\end{abstract}

Network based control of remote systems has been widely tackled, numerous methods are proposed to deal with small and/or constant delays. However, on long distance network (WAN) bandwidth and network delays may notably vary (beyond a few seconds) according to events occurring all along the transmission lines [OBO97]. On these asynchronous links the master slave relationship is also asynchronous; solutions not based on teleprogramming are fewer.

The paper presents the long distance remote control of our wheeled mobile manipulator; it is done through the use of different transmission media: wire $\left(\mathrm{WAN}^{1}\right)$ and radio links (WLAN ${ }^{2}$ 802.11 for instance). They both have a limited bandwidth and transmission delays on WAN fluctuate due to propagation, packetisation, distance, traffic, and others events.

The system can be remotely operated at two different levels: the operator request can correspond to high-level orders (tasks or sequence of tasks corresponding to a mission) which are then autonomously performed [CAR02], or to setpoints of a low-level remote control law for a "direct" teleoperation. The paper will focus on the latter.

Direct teleoperation lies on a generic teleoperation architecture (Figure 1) [LEL99]. The remote system includes the communication with the base (server block) and the local control of the robot state variables: velocity, steering and axes of the manipulator (controller block). On both sides (base and remote system), is included a compensation for the variations of transmission delays so that, on one hand the controller receives periodic setpoints and on the other hand the operator correctly perceives the remote system state (Prediction block associated with the Dynamic Model block) on its interface. Without this estimation, the more the mean delay grows, the more difficult it is for the operator to view what he is doing.

\footnotetext{
${ }^{1}$ Wide Area Network

${ }^{2}$ Wireless Local Area Network
} 


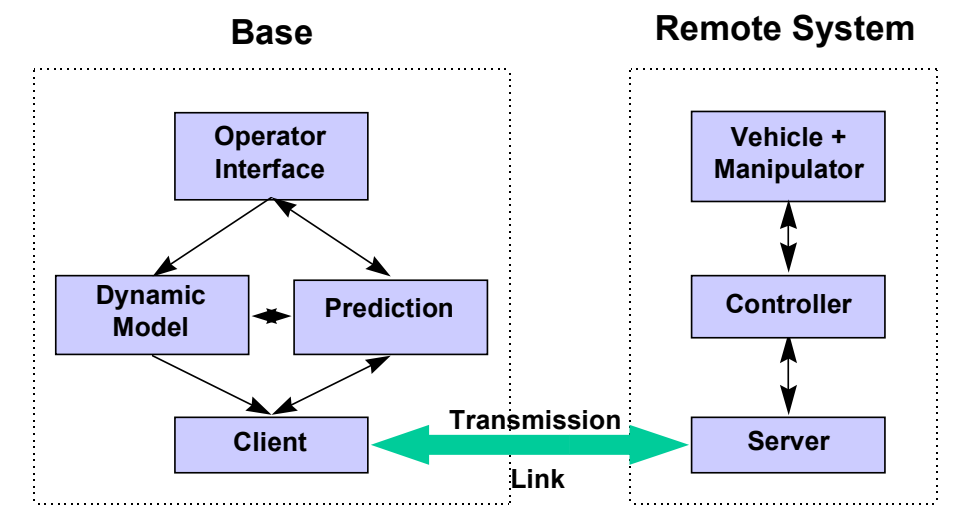

Figure 1 - Our teleoperation architecture

However, qualitative and quantitative results we obtained highlight limitations of our architecture when the delays vary too much or their mean value is too high, and when the estimations are too far from the reality [LEL01]. Out of the valid interval of compensation and estimation, the remote low-level control is not safe anymore (or less efficient when dealing with short networks as an intranet). Compensation and estimation are thus locally supervised, this remote supervisor being in charge to manage the system configuration and behavior in such cases. This work, briefly exposed below, will constitute the core of the full paper; we will expose our latest simulation results.

Events the local supervisor has to deal with can be of two major types: events due to the communication itself (architecture and protocols) and events due to the contents of the communication (setpoints values).

To compensate the varying time delays our system proceeds through a queue able to handle their effect until a maximum value. This queue stocks setpoints and delivers them at fixed step time to the embedded low-level control. The dimension of this queue is dynamically managed according to the network delays (measured). For example, in case of medium breakdown, maximum delay overflow, emptied queue, or queue overflow, the local supervisory controller will react. All those events will trigger an appropriate reaction as a dynamic adaptation of the queue when possible, or lastly a commutation to an autonomous mode ensuring a secure control of the robot until the re-establishment of satisfactory communication.

Moreover, when the operator directly pilots the robot (with a joystick for example) he sends requests containing setpoints for a low-level control loop; these setpoints may lead the robot into singular configurations for which the control becomes unstable. In such a case, the local supervisor automatically preempts this teleoperation and switches to a control mode able to face such singularities. Once the singular configuration has been crossed, it switches back to the previous teleoperation mode [CAR02].

The paper will present the architecture, the models and the principles of this remote supervised delay regulator. The behaviour of the system will be illustrated with simulation results. 


\section{References}

[CAR02] JD. Carbou, D. Andreu, P. Fraisse, "Events as a key of an autonomous robot controller", IFAC'02, Barcelona, Spain, July 21-26, 2002.

[OBO97] R. OBOE, P. FIORINI, "Internet based telerobotics: problems and approaches", Int. Conf. on Advanced Robotics (ICAR'97), pp 765-770, Monterey (CA-USA), July 1997.

[LEL99] A. Lelevé, P. Fraisse, P. Dauchez, F. Pierrot, "Teleoperation through the Internet: Experimental Results with a Complex Manipulator", $30^{\text {th }}$ International Symposium on Robotics (ISR), Tokyo, October 27-29, 1999.

[LEL01] A. Lelevé, P. Fraisse, P. Dauchez, "Telerobotics over IP Networks : Toward a lowlevel real-time architecture", Int. Conf. on Intelligent Robots and Systems (IROS'01), Maui, Hawai, USA, Oct. 2001. 